\title{
Study of Image Contrast Effects and Field Trends in Magnetic Recording Media by Static Magnetic Force Microscopy
}

\author{
Stefano Pergolini and Ugo Valdrè
}

Electron Microscopy Centre, Physics Department of the University and INFM-CNR, via Irnerio 46, 40126 Bologna, Italy

(Received March 17; accepted July 14, 1995)

\begin{abstract}
Co, Ni and Permalloy probes for Magnetic Force Microscopy have been characterized. The latter type of probe shows a particular behaviour which has been interpreted on the basis of the low coercivity of the Permalloy. The trend of the force due to the $z$-component of the stray magnetic field of digital recording media (Floptical) has been worked out, from the cantilever deflections, as a function of probe-sample distance and interpreted.
\end{abstract}

\section{Introduction}

Scanning Probe Microscopy (SPM), born with the invention of the Scanning Tunneling Microscope (STM) [1] and developed thereafter by the realization of the Atomic Force Microscope $(\mathrm{AFM})$ [2], is not only a powerful technique for the observation of surfaces at atomic resolution, but also a direct and non-destructive method for the detection of magnetic fields. STM has been successfully applied to image the Abrikosov fluxon lattice in type II superconductors and to measure other superconducting properties [3], whereas the Magnetic Force Microscope (MFM) [4-6], a derivation from the AFM, has been used to provide images of tracks in magnetic recording media and domain walls in ferromagnetic materials.

The present research work aimed at the setting of a MFM static technique, and the results obtained on magnetic recording media are given; they are the first step in the development of a method for the observation of magnetic structures in high $T_{\mathrm{c}}$ superconductors.

In addition, a description is given of the behaviour of probes made of various magnetic materials in order to optimize the MFM technique and its application to the investigation of the stray magnetic field emerging from a "Floptical" diskette.

\section{Materials and Methods}

The AFM used is a Digital Instruments NanoScope Multimode equipped with $\mathrm{Si}_{3} \mathrm{~N}_{4}$ pyramidal tips. They were coated, by evaporation, with layers of various thickness of $\mathrm{Co}, \mathrm{Ni}$ and Permalloy ranging from 20 to $70 \mathrm{~nm}$ (Fe has been discarded owing to its chemical instability). Values for 

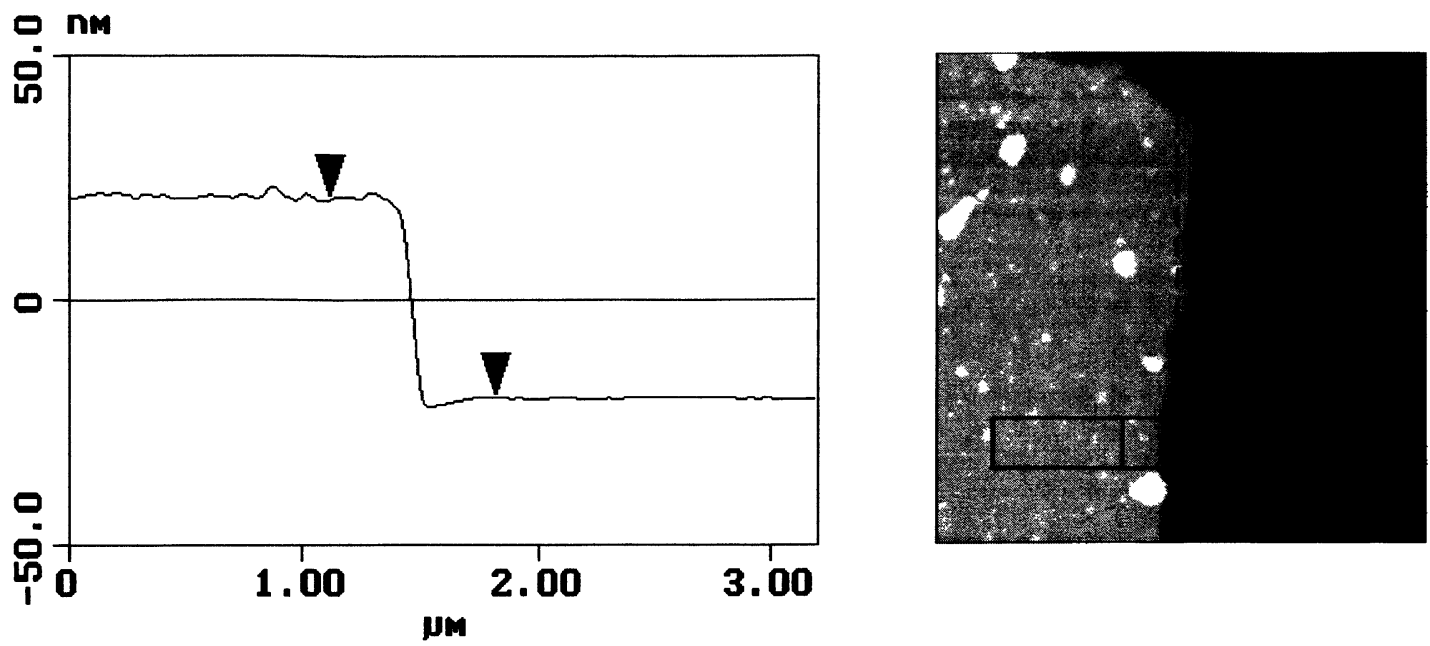

Fig. 1. - Section analysis of the step generated on the cantilever substrate by scratching away some of the deposited ferromagnetic material (permalloy in this case). The profile shown is obtained after averaging over the region defined by the black lines in the right hand side image. The sections are perpendicular to the dashed line, which has been chosen parallel to the step. The distance between the triangular markers along the vertical axis is $40.4 \mathrm{~nm}$.
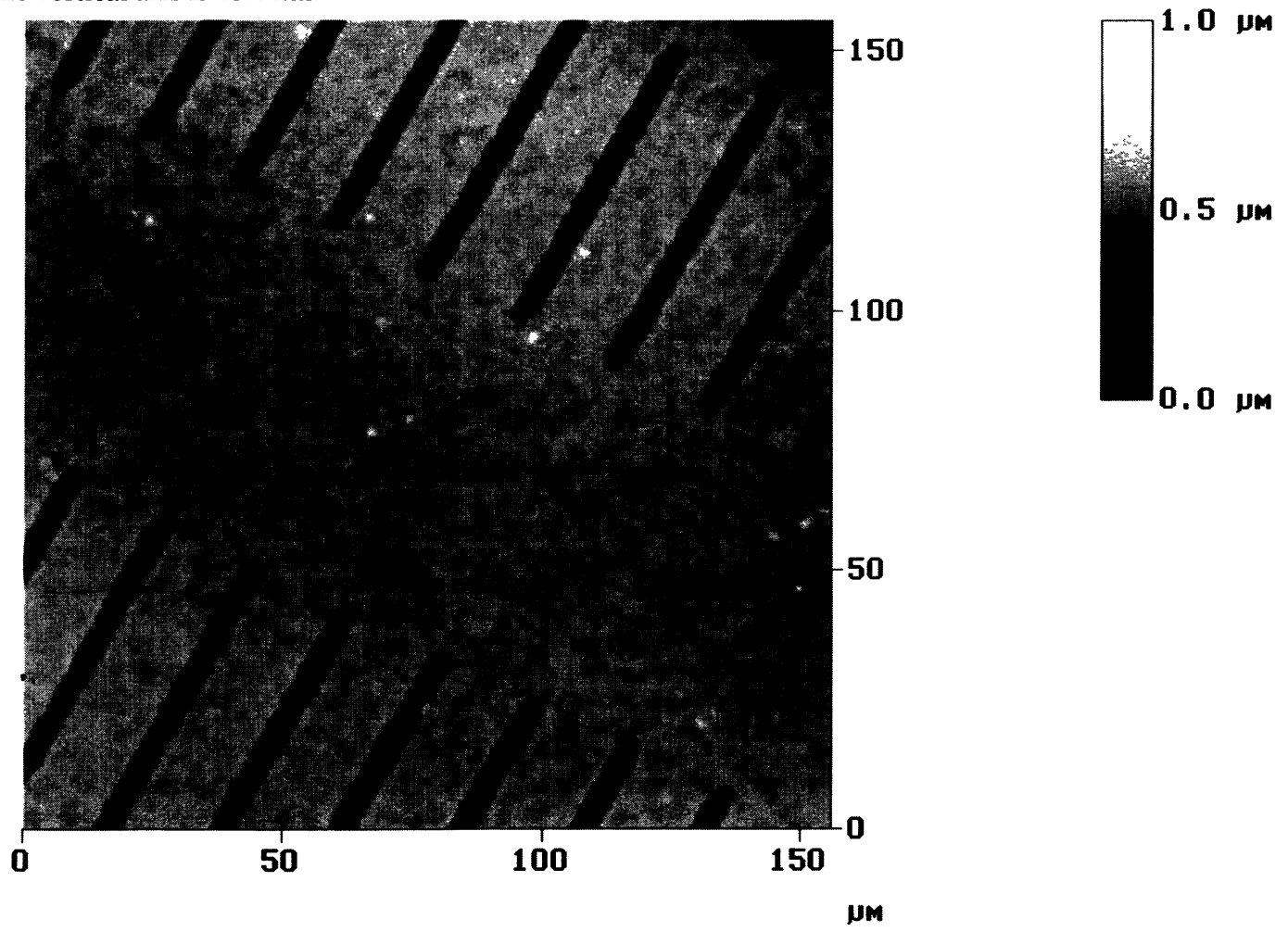

Fig. 2. - Topographic image of the sample (a "Floptical" diskette) acquired in the contact mode with a Digital Instruments "Stand Alone" AFM. The magnetic tracks, about $15 \mu \mathrm{m}$ wide, are located between the grooves (black stripes) used for the alignment of the readback head. 
the thickness have been directly obtained by the use of the AFM: some of the material deposited on the substrate of the cantilever has been gently scratched away and the height of the step so produced has been measured (Fig. 1). The accuracy is better than 5\%. Local and average values can be obtained by varying the size of the window indicated in Figure 1, where the average is performed.

The "Floptical" material is a hard magnetic alloy whose topographic details, visible in a low power optical microscope, are useful to locate the regions where to perform the AFM observation. A typical topographic AFM image is presented in Figure 2.

As stated above, the present work is finalized to the observation of superconducting magnetic structures where the use of the "Stand Alone" AFM is required. This instrument, however, cannot work in the dynamic (tapping) mode which would be the appropriate mode of operation for the study of magnetic fields, owing to its high sensitivity [4-6]. We have therefore used a static mode of operation, which consist in performing the scanning of the specimen by taking one line scan in the contact mode followed by a line scan at constant distance from the sample ("lift mode") over the same region. This allows the separation of the magnetic contrast from the topographic features [6] as shown in Figure 3.

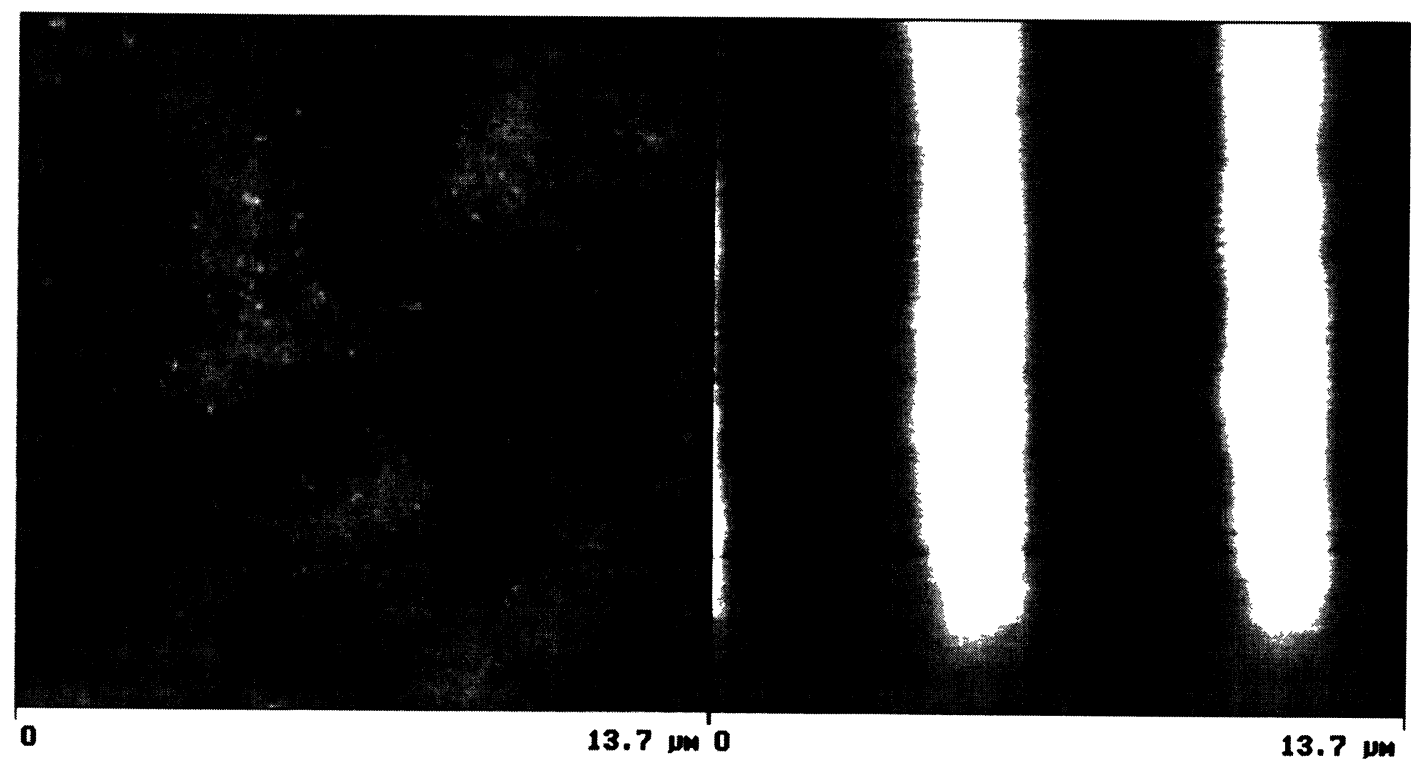

Fig. 3. - Topographic image taken in the contact mode (left) and magnetic force image obtained in the "lift mode" (right) with a Ni probe. The specimen is a "Floptical" diskette. The dark and bright regions are the borders of the magnetic marks, where the $z$-derivative of the $H_{z}$ component of the stray magnetic field reaches its maximum or minimum value. The marks are approximately $15 \mu \mathrm{m}$ long and $3 \mu \mathrm{m}$ wide.

\section{Results}

3.1 Comparison BetweEn MAgnetic Probes - AFM images acquired with both Co and Ni probes show a regular sequence of attractive and repulsive regions, which reproduce the trend of 
the $z$-derivative of the $H_{z}$ component of the sample stray magnetic field (Fig. 4). The length, $L$, of the magnetic mark is $3.0 \mu \mathrm{m}$. The maximum cantilever deflection is $3.5 \mathrm{~nm}$ in both directions (up and down) for the Co and $3.8 \mathrm{~nm}$ for the Ni probes. It should be noted that the practical coincidence of the cantilever deflections is casual, since the deflections depend on several parameters (e.g., elastic constant of the cantilever, magnetic moment of the probe), some of them out of the operator control.
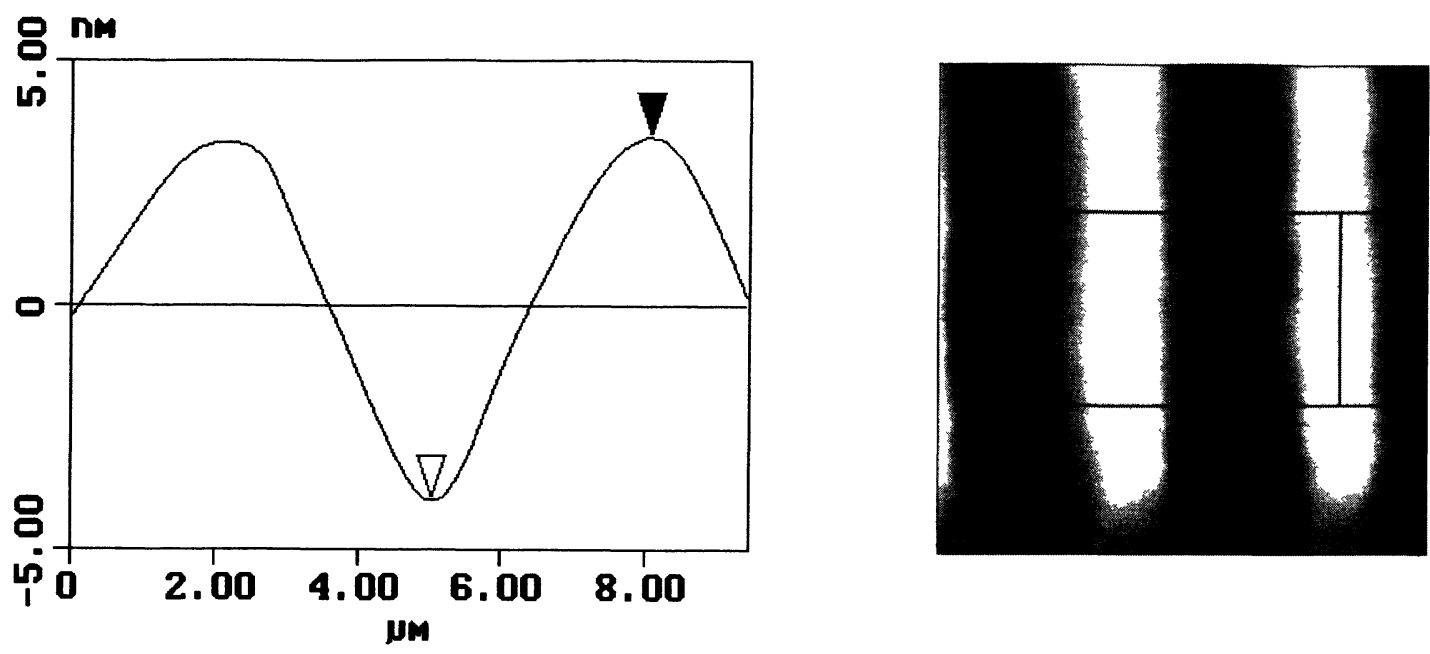

Fig. 4. - Section analysis of the contrast generated by the stray magnetic field from a "Floptical" diskette detected with a Co probe. The horizontal distance, $L$, between the white and black triangular markers gives the width of the mark; the vertical distance is the maximum deflection of the cantilever.

As for the Permalloy, the AFM images show a peculiar trend (Fig. 5). From right to left we note a repulsive region which reaches a narrow maximum, followed by a small depression, then by another maximum of amplitude, smaller than the previous one. Finally, the probe enters into the attractive region and the deflection shows a trend similar to that of Co and Ni. This is true irrespective of the chosen track; observations performed on the same track with the various types of tip have confirmed the above statement.

The results obtained with the Permalloy probe can be understood as follows. Given the low coercivity of the FeNi alloys, the repulsive stray field from the "Floptical" acting on the tip reaches a value, during scanning, capable of altering the magnetization of the probe; this reduces the $z$-component of its magnetic moment and gives rise to the shallow minimum. As the scanning motion proceeds, the probe reaches the region where the repulsive field has a maximum and then enters into the attractive region, where the original magnetization is restored. To be noted that the total magnetic moment of the probe is practically directed along $z$, irrespective of the distance probe-sample, owing to the symmetry of the tip. Further evidence of the above interpretation is given by taking the profile on reversing the scan direction: the observed profile is again asymmetrical, but in the opposite direction of the abscissa axis.

We have tried to give a quantitative basis to the previous reasoning by making two assumptions.

(i) The deflection, $D(x)$, of the cantilevers carrying the Co and Ni probes reproduces faithfully the trend of the $z$-derivative of the stray magnetic field of the sample. This trend is well 

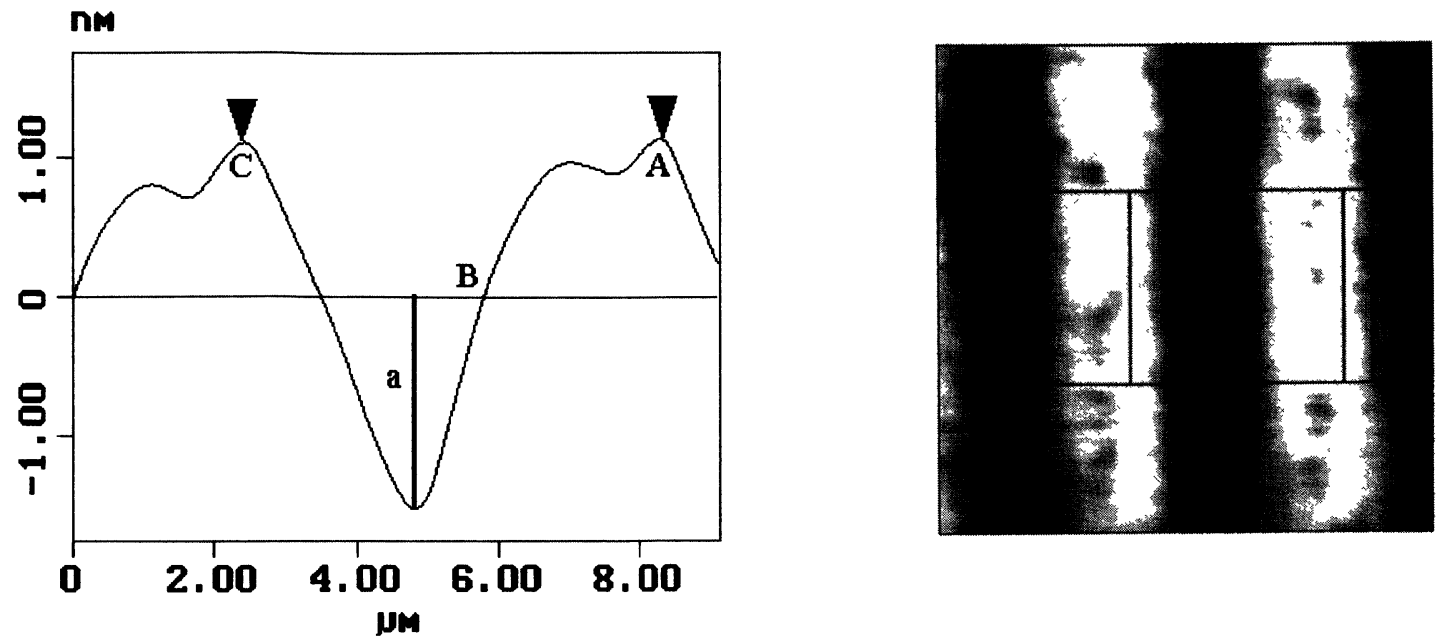

Fig. 5. - Section analysis of the contrast generated by the stray magnetic field emanated by a "Floptical" sample. A Permalloy probe was used. The peculiar trend can be explained in terms of the probe-sample interaction which alters the magnetic moment of the probe. Note that the horizontal distance between the black triangular markers (A and $\mathrm{C}$ ) is the period of the structure, which is twice the mark width $L$.

approximated by a sine function

$$
D(x)=a \sin \frac{2 \pi x}{2 L}
$$

as seen from Figure $4(2 L$ is the profile spatial periodicity and $a$ is a constant depending on the probe and on the cantilever).

(ii) The value of the magnetization of the permalloy probe changes (rather abruptly, at the points indicated in Fig. 5), so that, $D(x)$ is given by:

$$
D(x)=h(x) \sin \frac{\pi x}{L}
$$

$h(x)$ must be a periodic function having the same period $2 L$ of equation (1). The trend is consistent with a hysteresis phenomenon. For the specific probe used, by taking $h(x)=a$ (the maximum cantilever deflection in the attractive regime) between points $\mathrm{B}$ and $\mathrm{C}$ and $h(x)=a / 2$ between $\mathrm{A}$ and $\mathrm{B}$, a reasonable agreement is found with the experimental profile.

3.2 AnAlysis of The $H_{z}$ Component of the STRAy Magnetic Field - The trend of the $H_{z}$ component can be plotted by scanning the probe at various heights from the sample surface. The measurements have been performed within the range of $50 \mathrm{~nm}$ to $2 \mu \mathrm{m}$; the lower limit is set by the specimen surface irregularities (of the order of $100 \mathrm{~nm}$ ), whereas the upper limit is set by the maximum traverse of the $z$-piezo drive. The scanning level can be changed without breaking the tip-specimen engage. A series of magnetic images have been taken, each image at a different level. Typical images are shown in Figures 6a, b, c, d (taken with a FeNi tip). Each image profile has been analysed; the difference between the maximum and the minimum (see, e.g., Fig. 5 left) has been measured and plotted in the graph of Figure 7 as a function of the scanning level. The experimental error is about $0.2 \mathrm{~nm}$. The fitting of these data in terms of a simple assumption (e.g. dipole-dipole interaction) [5] failed. In fact, on varying the tip-sample distance, various types 


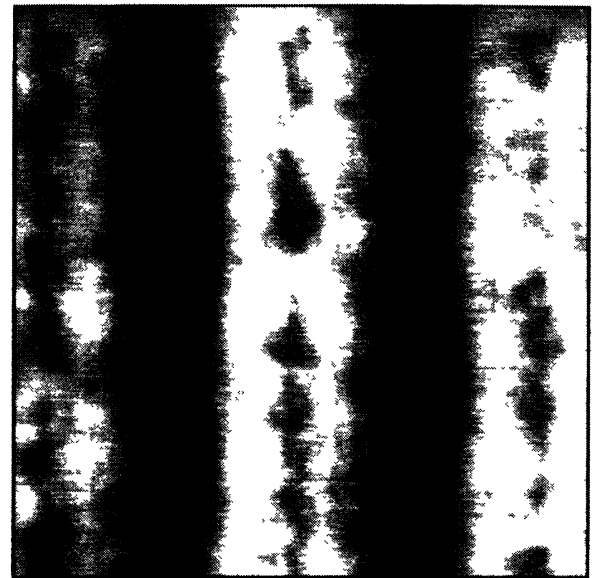

a

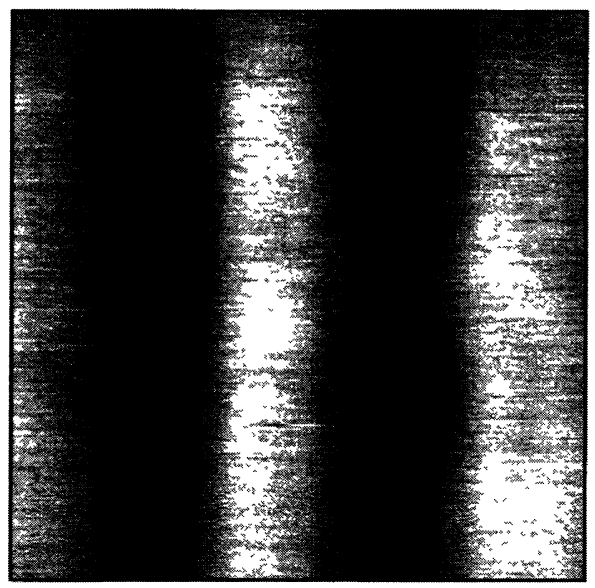

C
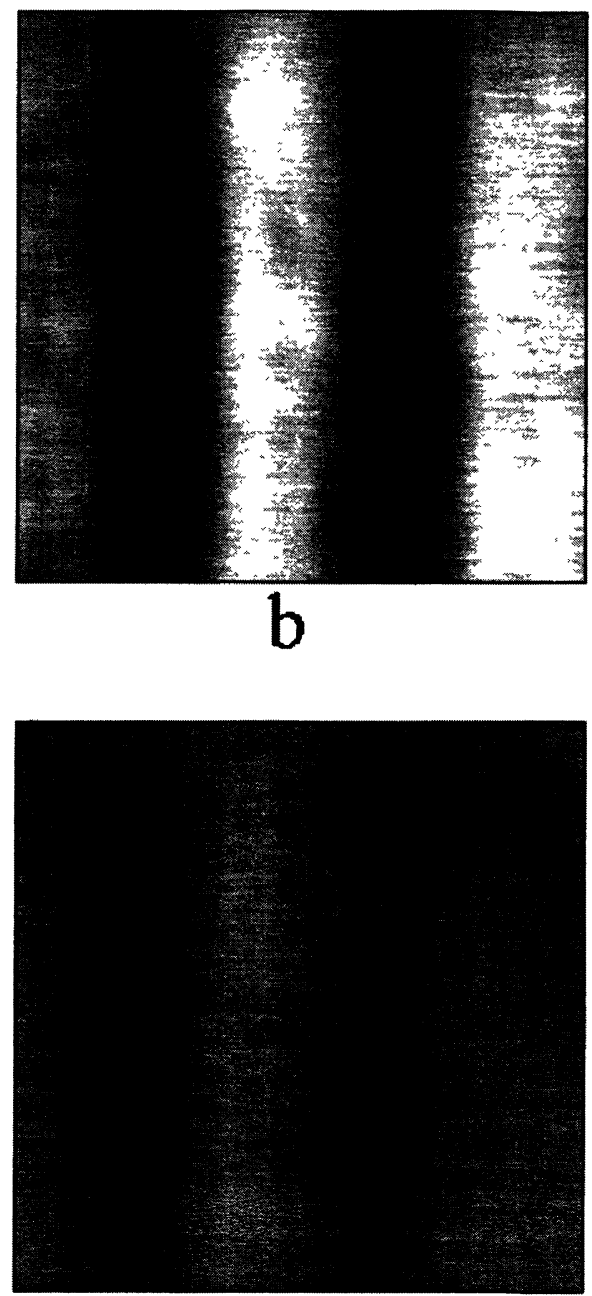

d

Fig. 6. - Typical images of the contrast generated by the stray magnetic field from the "Floptical" captured at various scanning heights (a: 50, b: 300 , c: 400 , d: $2000 \mathrm{~nm}$ ) with a Permalloy-coated tip. Note that the shallow minimum, located in the middle of the repulsive region (well visible in Fig. 6a), disappears around $400 \mathrm{~nm}$.

of interaction are possible. At small distances $(200-500 \mathrm{~nm})$ a strong dipole-dipole interaction is experienced; at larger distances the repulsive monopole-monopole interaction prevails; this is consistent with the results of Figures $6 \mathrm{a}, \mathrm{b}, \mathrm{c}$, d. At distances greater than about $1000 \mathrm{~nm}$ the bulk effect of the whole cantilever and specimen system occurs, together with instrumental effects (e.g., piezo-drive hysteresis, lack of linearity of the cantilever deflection). The latter is also present below about $200 \mathrm{~nm}$. The curve of Figure 7 represents the best fit. 


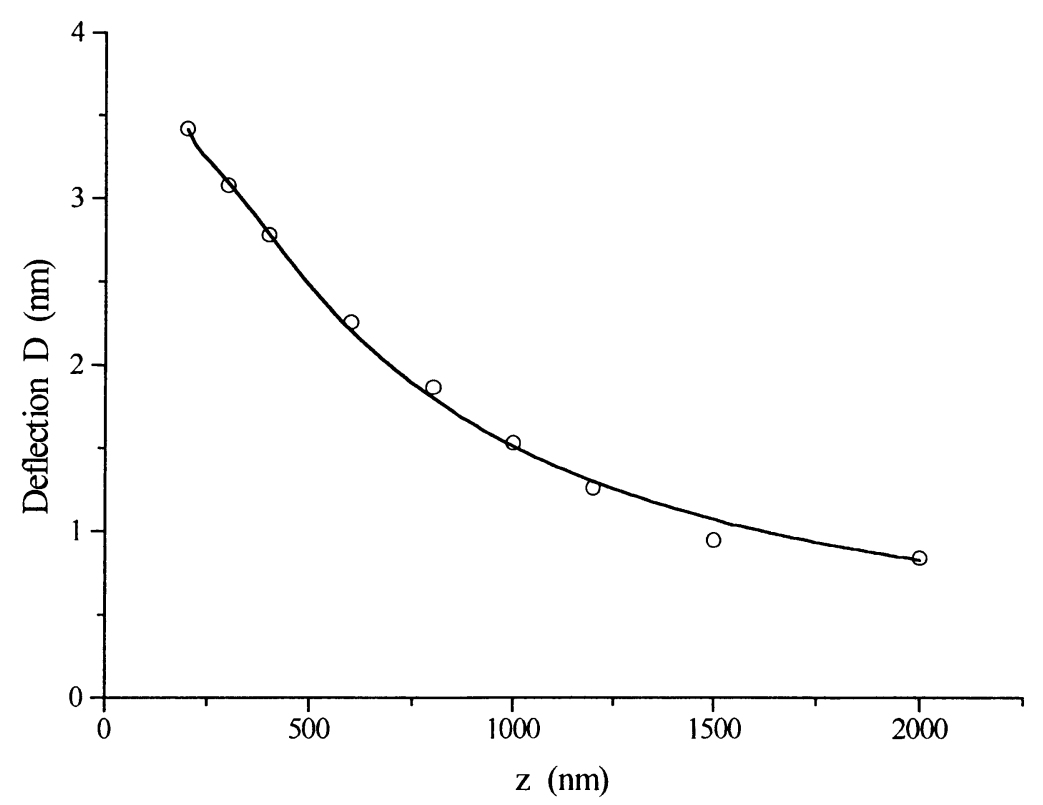

Fig. 7. - Experimental (hollow circles) and theoretical (continuous line) trends of the maximum cantilever deflection due to the magnetic interaction as a function of the scanning level $z$. The two trends agree down to $200 \mathrm{~nm}\left(\chi^{2}=0.004\right)$.

\section{Conclusions}

Cantilevers coated with $\mathrm{Co}, \mathrm{Ni}$ and Permalloy for Magnetic Force Microscopy have been characterized. The performances of the various probes have been assessed in terms of their suitability for the study of magnetic samples. Co and Ni probes faithfully reproduce the profile of the $z$ derivative of the stray magnetic field generated by hard, digital, magnetic recording media. The magnetic image contrast shown by Permalloy probes, when used with the above type of recording media, has been phenomenologically interpreted on the basis of the low coercivity of Permalloy.

In addition, the trend of the deflection $D$ produced by the $z$-component of the stray magnetic field, $H_{z}$, of recording media has been worked out as a function of probe-sample distance (the measure of the actual values of the magnetic field requires a calibration). A dipole-dipole interaction dominates the short distance forces $(200-400 \mathrm{~nm})$, whereas a repulsive monopolemonopole interaction prevails above about $400 \mathrm{~nm}$. Below $200 \mathrm{~nm}$ there are discrepancies between experimental trend and theoretical curve, which may be explained by the loss of linearity of the cantilever. This is not surprising since the cantilever is bent down by the stress produced by the evaporated magnetic film with respect to its original (pre-evaporation) resting position; hence the cantilever works under large deflection conditions (several $\mu \mathrm{m}$ ).

\section{Acknowledgements}

This work has been partly supported by MURST, Rome, and by the European Union. 


\section{References}

[1] Binnig G. and Rohrer H., Helv. Phys. Acta 55 (1982) 726.

[2] Binnig G., Quate C.F. and Gerber Ch., Phys. Rev. 56 (1986) 930.

[3] Hess H.F., Robinson R.B., Dynes R.C., Valles J.M. Jr and Waszezak J.V., Phys. Rev. Lett. 62 (1989) 214.

[4] Grutter P., MSA Bull. 24 (1994) 416.

[5] Rugar D., Mamin H.J., Guethner P., Lambert S.E., Stern J.E., McFadyen I.R. and Yogi T., J. Appl. Phys. 68 (1990) 1169.

[6] Babcock K., Photo. Spec. (May 1994) 1. 